\title{
Nanostructured surface fabricated by laser interference lithography to attenuate the reflectivity of microlens arrays
}

\section{P.-Y. Baroni}

pierre-yves.baroni@epfl.ch

\section{B. Päivänranta}

birgit.paivanranta@joensuu.fi

\section{T. Scharf}

toralf.scharf@epfl.ch

W. Nakagawa

wataru.nakagawa@ece.montana.edu

\section{Roussey}

matthieu.roussey@epfl.ch

\section{Kuittinen}

markuu.kuittinen@joensuu.fi

H. P. Herzig

hanspeter.herzig@epfl.ch
Optics and Photonics Laboratory, Institute of Microengineering (IMT), Ecole Polytechnique Fédérale de Lausanne (EPFL), A.-L. Breguet 2, 2000 Neuchâtel, Switzerland

Department of Physics and Mathematics, University of Joensuu, Joensuu, 80100, Finland

Optics and Photonics Laboratory, Institute of Microengineering (IMT), Ecole Polytechnique Fédérale de Lausanne (EPFL), A.-L. Breguet 2, 2000 Neuchâtel, Switzerland

Electrical and Computer Engineering Dept., Montana State University, Bozeman, MT 59717-3780, USA

Optics and Photonics Laboratory, Institute of Microengineering (IMT), Ecole Polytechnique Fédérale de Lausanne (EPFL), A.-L. Breguet 2, 2000 Neuchâtel, Switzerland

Department of Physics and Mathematics, University of Joensuu, Joensuu, 80100, Finland

Optics and Photonics Laboratory, Institute of Microengineering (IMT), Ecole Polytechnique Fédérale de Lausanne (EPFL), A.-L. Breguet 2, 2000 Neuchâtel, Switzerland

A subwavelength-scale square lattice optical nanostructure is fabricated using an interference photolithography process on the surface of a quartz microlens array. This nanostructuring of the quartz surface introduces an antireflective effect, reducing reflectivity between $10 \%$ and $30 \%$ and enhancing the transmissivity $3 \%$ in the visible spectrum. This approach permits fast fabrication on a 4 -inch wafer covered with microlenses (non-flat surface) and produces monolithic devices which are robust to adverse environments such as temperature variations. [DOI: 10.2971/jeos.2010.10006]

Keywords: antireflective, nanostructure, microlens, interference photolithography

\section{INTRODUCTION}

Fabrication of microlens arrays has been extensively explored [1]-[3] as they are widely used in a large range of applications, often to link between macro and micro optical components [4]. Antireflective (AR) coatings and surface treatments on microlenses have been proposed for different materials and wavelengths [5]-[7] and theoretical analyses have been performed [8]. The principal solution proposed in a number of papers consists of an antireflective coating deposited on the structure. Although this method is quite evolved, there are some applications where having a monolithic material structure is advantageous, such as in extreme temperature conditions or particularly aggressive environments, as presented in [9] for planar surfaces. Gratings on microlenses in plastic materials have been demonstrated using replication by hot embossing [10].

In this paper, we investigate an alternative approach. It consists of nanostructuring the surface of the microlenses, producing an AR layer having engineered effective refractive index properties which reduces the reflectivity and enhances the light transmission through the lens. In this work we demonstrate the feasibility of fabricating such nanostructures on a large, non-flat surface. In the following, we will present experimental results on such nanostructured monolithic lenses, which, although not ideal, exhibit significant AR characteristics.

\section{FABRICATION}

To begin, we describe the fabrication of a subwavelength AR layer on the surface of a quartz microlens array with interference photolithography and plasma etching. The challenge is to obtain an homogenous structure on the entire surface of each lens and on a large array of microlenses. The shape of the nanostructures we produce with this method is not as rectangular as the structures often studied. However, these more rounded, non-rectangular nanostructures still possess significant AR properties. Moreover, it allows the production of nanostructures quickly, inexpensively, and on large areas.

First, an hexagonal array of $145 \mu \mathrm{m}$ diameter microlenses is structured by photolithography and a reflow process [11] on a 4-inch quartz wafer. Then, the microlenses, which are $14 \mu \mathrm{m}$ high, are transferred into the quartz by Reactive Ion Etching (RIE). The pressure is set at 2.7 mbar and the flow rate is set at $3 \mathrm{sccm}$ (standard cubic centimeters per minute) of Ar, $20 \mathrm{sccm}$ of $\mathrm{O}_{2}$ and $34 \mathrm{sccm}$ of $\mathrm{C}_{3} \mathrm{~F}_{8}$ for an etching rate of approximately $300 \mathrm{~nm}$ per minute. 


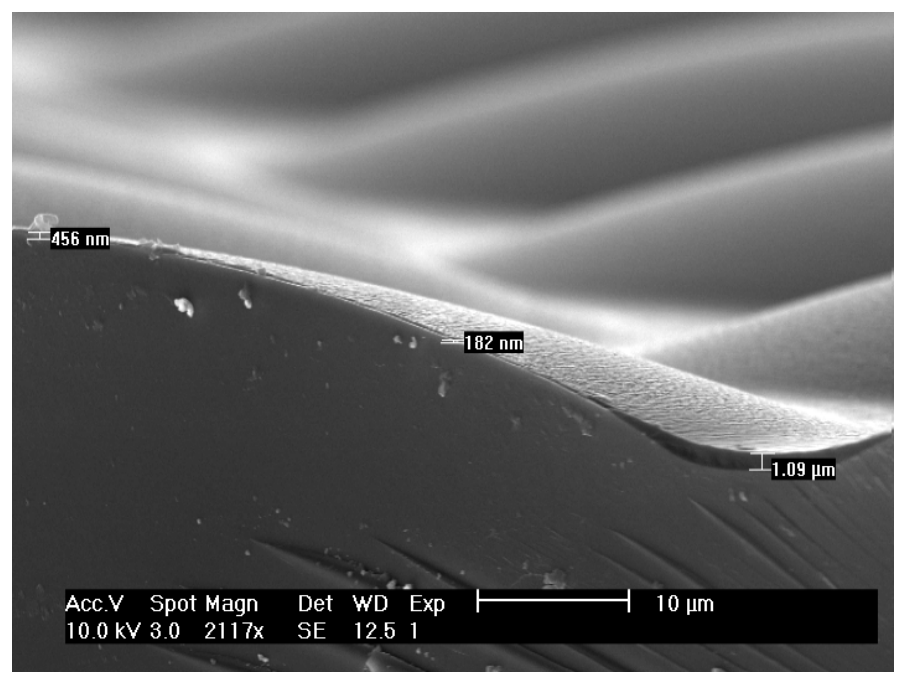

FIG. 1 Side view of a microlens with nanostructured photoresist shows that photoresist covers the entire surface of the microlenses, with varying thickness depending on the position and on the local slope.

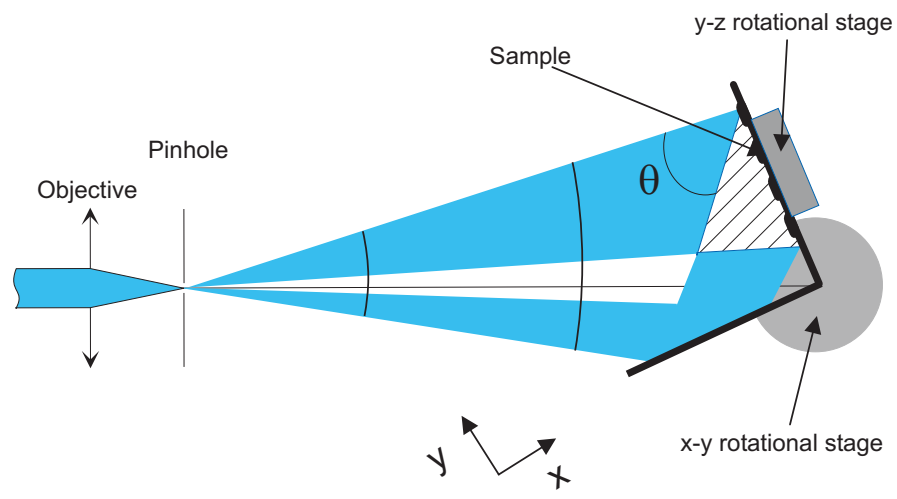

FIG. 2 Illumination with interference fringes created by superposition of direct and reflected light emanating from a single pinhole.
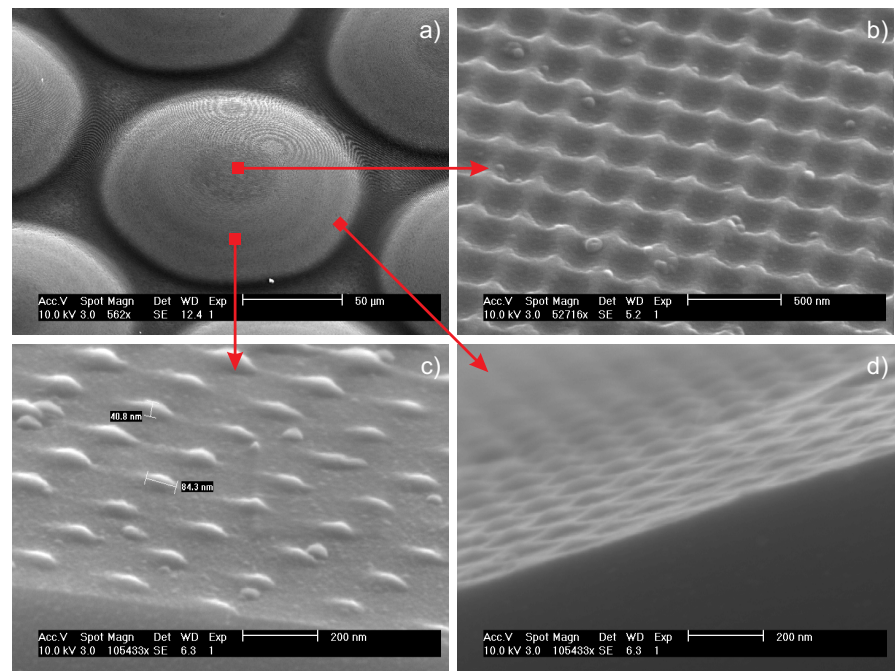

FIG. 3 (a) SEM picture of a $145 \mu \mathrm{m}$ diameter microlens with the entire surface covered by a square array of nanopillars. SEM pictures with higher magnification of different part of the microlens: (b) top region, (c) maximum slope region, and (d) edge region.

For the AR nanostructured surface, a $400 \mathrm{~nm}$ diluted photoresist (PR) is spun at very low speed (400 rpm) for 25 seconds, in order to cover fully the surface of the microlenses. The Scanning Electron Microscope (SEM) picture shown in Figure 1 presents a side view of a microlens coated by the nanostructured PR. One can see that due to the slope induced by the microlens' curvature, the height of the photoresist coating is not uniform over the entire surface [12]. Indeed, the PR thickness varies between the lower $(\sim 1 \mu \mathrm{m})$ and the upper $(\sim 450 \mathrm{~nm})$ parts of the microlenses with a minimum around $200 \mathrm{~nm}$ where the slope is greatest. This requires that the exposure parameters for the photolithography be set for the thinnest part of the resist in order to avoid overexposure.

After a prebake at $40^{\circ} \mathrm{C}$ and $50^{\circ} \mathrm{C}$ for thirty minutes each, the nanostructure pattern is introduced by interference photolithography [13]. A UV laser beam $\left(\mathrm{Kr}^{2+}\right)$ is expanded and spatially filtered passing through a microscope objective (20X) and a $10 \mu \mathrm{m}$ pinhole to create a coherent spherical wavefront (see Figure 2), which can be approximated in the far field as a planar wavefront for a small area. The fringes are created by placing a mirror at $90^{\circ}$ with respect to the sample (Lloyd's mirror configuration). The grating period, $\Lambda$, is selected by adjusting the angle, $\theta$, between the two incident beams using the $x-y$ rotational stage following the equation $\Lambda=\lambda / \sin \theta$, where the laser wavelength, $\lambda$, is fixed at $413 \mathrm{~nm}$ [14]. Then the wafer is rotated in the $\mathrm{y}-\mathrm{z}$ plane by $90^{\circ}$ and exposed again to obtain a two dimensional square lattice.

The power on the sample is set at $1 \mu \mathrm{W} / \mathrm{cm}^{2}$ and the total exposure time is approximately one minute.

After a post baking process at $80^{\circ} \mathrm{C}$, the last step is to transfer the nanostructures from the resist into the quartz. This is achieved using a short plasma etch $\left(30 \mathrm{sccm} \mathrm{C}_{3} \mathrm{~F}_{8}+1 \mathrm{sccm}\right.$ Ar). The maximum thickness of quartz removed in this step is $200 \mathrm{~nm}$, so the shape of the microlenses is only minimally affected. After removing the photoresist, we obtain a 4-inch wafer covered with microlenses having a nanostructured AR surface in quartz.

Figure 3 shows SEM pictures of a nanostructured microlens (see Figure 3(a)) and close-up views of the shape of the nanostructures on different areas of the lens (see Figures 3(b), 3(c) and $3(\mathrm{~d}))$.

The annular rings (Moiré effect) observed in the SEM picture in Figure 3(a) show that the nanostructures completely cover the non-flat surface consisting of microlenses. The periodicity is $270 \mathrm{~nm}$ in both transverse directions, independent of the position on the lens.

The nanostructures in the central region of the lens (see Figure 3(b)) are approximately $100 \mathrm{~nm}$ deep, and have a slightly rounded shape as compared to the rectangle structures studied theoretically [5]. In addition, one can observe some links between the pillars. In the maximum slope region (see Figure 3(c)), we can see that the links between the pillars have disappeared, and the structures are approximately $40 \mathrm{~nm}$ deep. In the edge region of the lens (see Figure 3(d)), the shape of the nanostructures have the same profile as in the central region (see Figure 3(b)). The variations in the aspect ratio of the nanostructures are due to the inhomogeneity of the PR thickness as shown in Figure 1. 


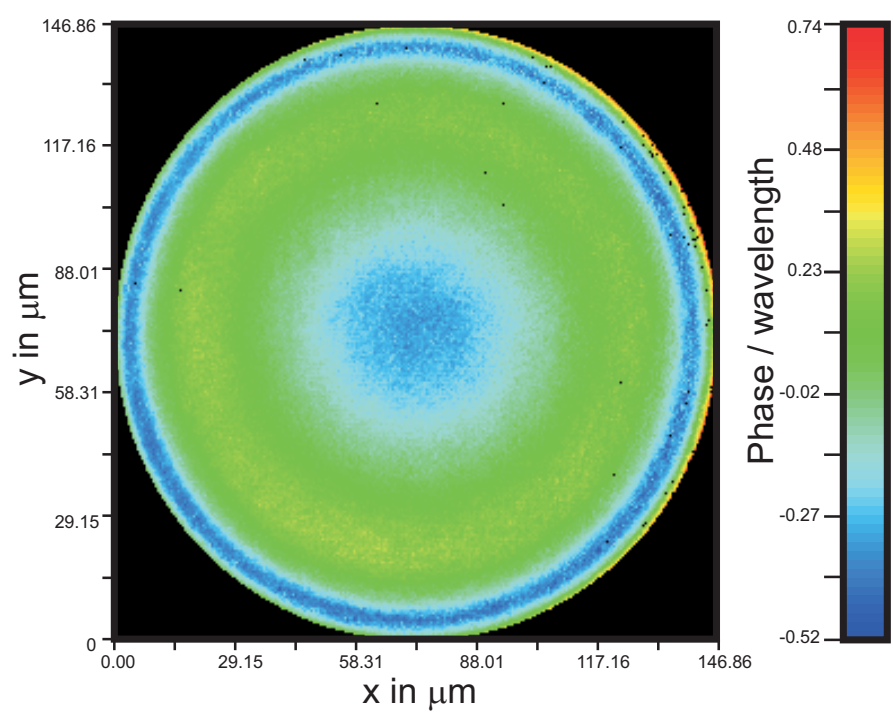

FIG. 4 Measured phase image of a nanostructured microlens using an interference microscope, working at $632 \mathrm{~nm}$ wavelength, to characterize the Strehl ratio.

Note that the non-rectangular nanostructure profile does not, a priori, impact the desired properties of our device, as our goal is not to obtain a wavelength filter but rather a broadband AR effect. An optimization of the PR deposition process shall be envisaged to improve the homogeneity. Nevertheless, as we will demonstrate, these nanostructures result in a significant reduction in the reflectivity of the microlens surface.

The quality of the microlenses is measured using a MachZehnder interferometric microscope, using an iris to select a single lens [15]. The phase and intensity of the interference pattern are measured, then the Strehl ratio of the lenses is calculated. The Strehl ratio is the ratio between the peak diffraction intensities of an aberrated versus a perfect wavefront. Conventionally, the acceptable value for the Strehl ratio is greater than 0.8 , which corresponds to an rms wavefront error of $\lambda / 14$ (Marechal's criterion [16]). For the quartz microlenses without nanostructure, we obtain Strehl ratios between 0.92 and 0.99 depending on the specific lens selected. After the plasma etching, due to the PR thickness variation, the shape of the microlenses changes slightly and the Strehl ratio is measured to be around 0.9. The measurements are done on different areas of two wafers of each type. From the phase image shown in Figure 4, we observe that the aberrations come mostly from the outer part of the lens.

From these measurements, we demonstrate the feasibility of producing a nanostructured AR surface on a quartz microlens array without significantly reducing the quality of the lenses. Indeed, the challenge here is to fabricate a combination of micro- and nano-scale structures on a large surface in the same material.

\section{CHARACTERIZATION IN REFLECTION}

In the previous section, we demonstrated the production of a microlens array with its entire surface covered by a nanostructured AR square grating. However, the fabricated nanostructures were non-ideal in several respects, including vari-

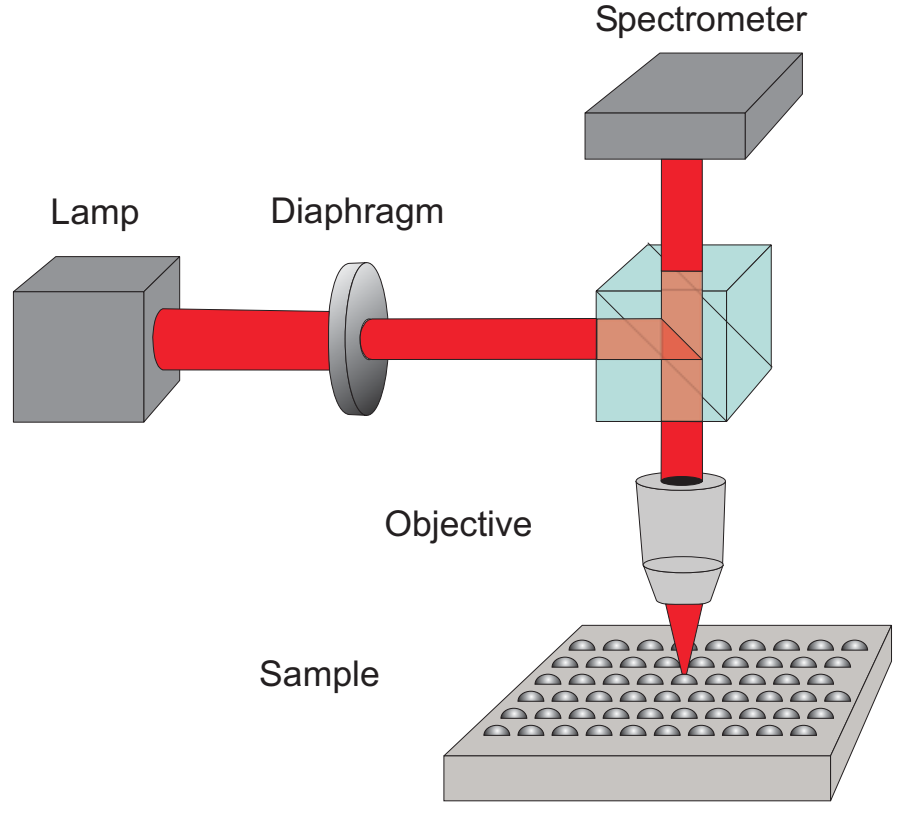

FIG. 5 Schematic drawing of the setup for reflectivity measurements of different samples. The light from a halogen lamp is focused onto the sample, and the reflected light is then collected to be analyzed by a spectrometer.

ability in the nanostructure size depending on the position on the lens, and deviation of the pillar shape from the rectangular profile of the original design [5]. In this section, we will show through experimental characterization that these nonideal nanostructures still provide a significant attenuation of the reflectivity of the microlens array.

The setup used for the reflection measurements is shown schematically in Figure 5. It is based on a standard microscope stand (Leica DMR) with a spectrometer (Ocean Optics 2000) mounted at the end of the light path. Three objectives have been used: $100 \times(\mathrm{NA}=0.9), 40 \times(\mathrm{NA}=0.6)$ and $20 \times(\mathrm{NA}$ $=0.4$ ). An adjustable circular diaphragm is added in the light path to be imaged onto the sample. It allows control of the spot size to be measured. This setup will be used for all reflection measurements of our samples.

The first step is to characterize the performance of the nanostructured AR surface on a flat substrate. A flat quartz wafer without nanostructures is measured to obtain a reference signal $\left(R_{\text {ref }}\right)$ with the image of the diaphragm is set at $140 \mu \mathrm{m}$ diameter. Then, the reflection of the nanostructured sample is measured $(R)$ with the same parameters of illumination and detection. The graph shown in Figure 6 shows the ratio between the spectral response of the nanostructured surface and the reference signal. The red curve is the result using a $100 \times$ objective with a numerical aperture of 0.9 and the black curve using a $40 \times$ objective with a numerical aperture of 0.6 . The reflectivity is decreased between 10 and $35 \%$ with the $100 \times$ objective and between 5 and $10 \%$ with the $40 \times$ objective. This means that the nanostructure layer has an antireflective effect over the entire visible wavelength range, as was predicted theoretically and already demonstrated experimentally with other materials [5].

Furthermore, using a higher numerical aperture (i.e. a larger 


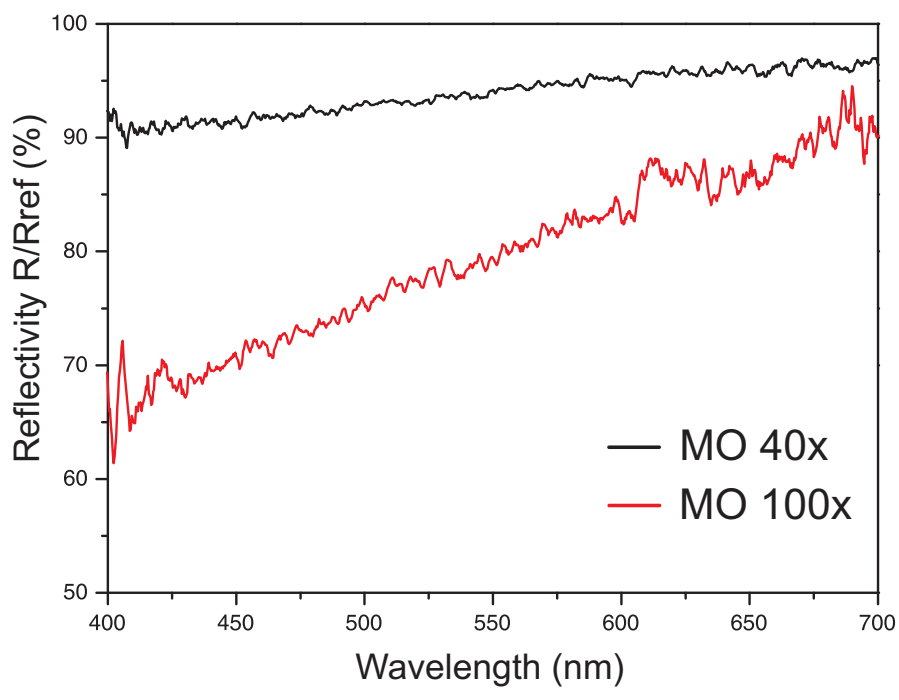

FIG. 6 Reflectivity spectra of the AR nanostructures on a flat substrate normalized by the reflectivity of a flat non-structured wafer, using a 100x objective, NA $=0.9$ (red) and a $40 \times$ objective, NA $=0.6$ (black).

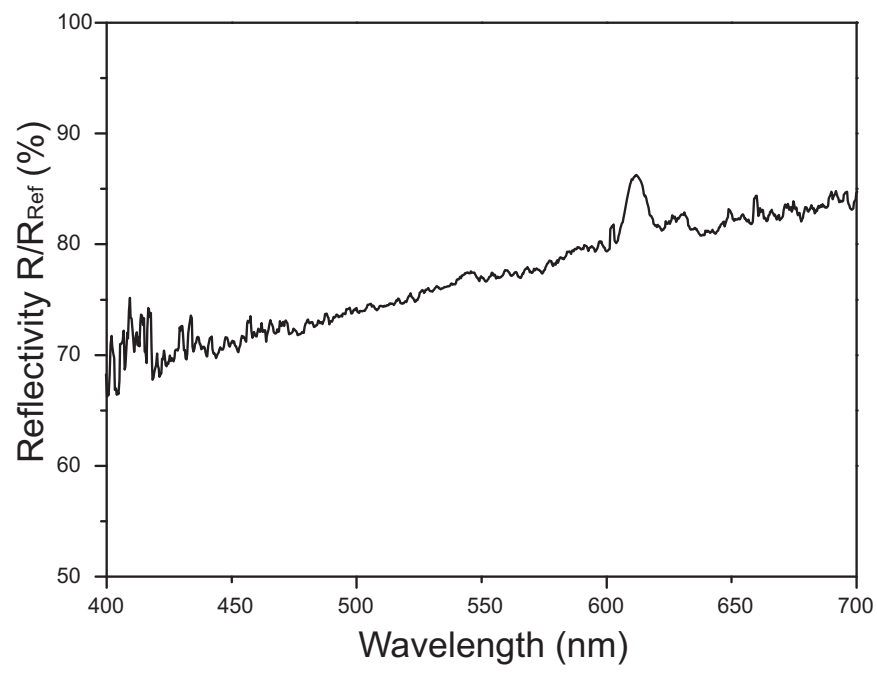

FIG. 7 Normalized reflectivity spectrum for an area containing several nanostructured microlenses.

illumination angle) reduces the reflectivity, which qualitatively agrees with the theoretical predictions presented in Table 2 of [5].

The reflectivity varies as a function of the wavelength, as demonstrated in [17]. In the results shown in Figure 6, the largest attenuation is obtained for smaller wavelengths. Theoretically, the minimum of reflection should be centered around $550 \mathrm{~nm}$. However, since the height of the nanopillars is lower than in the design, it is to be expected that this minimum is shifted to lower wavelengths.

Moreover, measurements are made on different areas of the sample with essentially identical results (less than $5 \%$ variation). This shows that the nanostructures are relatively homogenous over the entire 4-inch wafer.

From these results, we have demonstrated that we are able to fabricate a square lattice of nanopillars with a periodicity

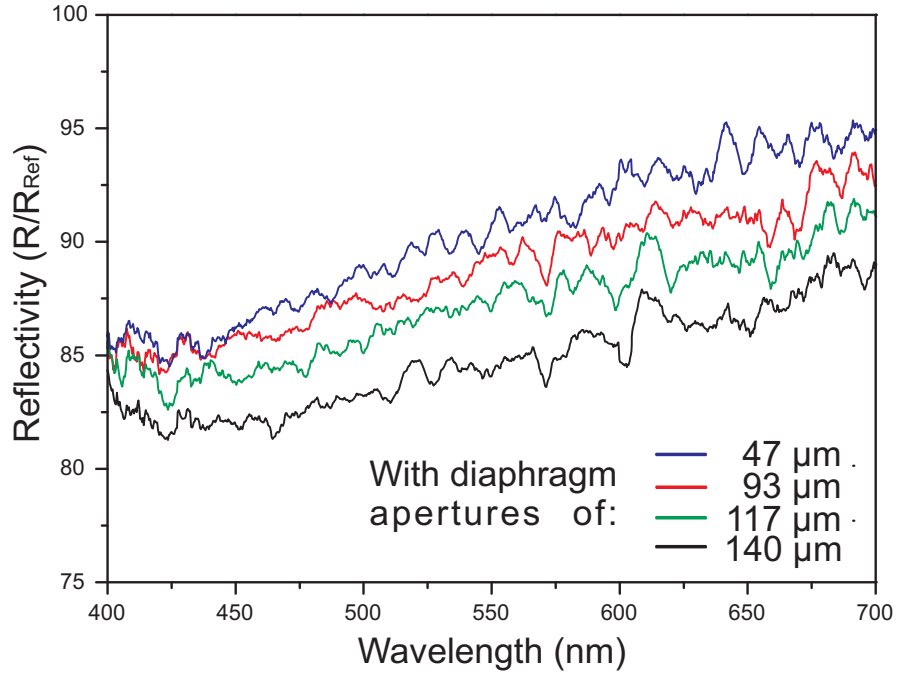

FIG. 8 Reflectivity ratio between lenses with and without AR nanostructures for aperture diameters of $47 \mu \mathrm{m}$ (blue), $93 \mu \mathrm{m}$ (red), $117 \mu \mathrm{m}$ (green) and $140 \mu \mathrm{m}$ (black). The reflectivity is decreased between $5 \%$ and $14 \%$ over the visible spectrum.

of $270 \mathrm{~nm}$ on a 4-inch wafer. A non-negligible attenuation of the reflectivity has been observed and could be improved by producing taller nanostructures.

The objective is then to determine whether the nanostructures maintain the same AR behavior on a microlens array instead of on a flat surface. The setup shown in Figure 5 is used to characterize these samples. In this case the reference is defined by measuring the reflectivity of an array of quartz microlenses without AR nanostructures, and all the results are normalized by this reference.

Two different studies have been performed. The first consists of the measurement of the reflectivity of a surface containing several nanostructured microlenses $(\sim 7)$. In this case, a $20 \times$ objective with a numerical aperture of 0.4 is needed to image a large enough area ( $450 \mu \mathrm{m}$ in diameter). For both the sample and the reference, the center of one microlens is positioned in the middle of the light path. The results are shown in Figure 7.

The attenuation of the reflectivity is between $30 \%$ and $15 \%$ over the visible spectrum. The curve shown is an average of two measurements acquired on two different areas of the sample.

The second experiment measures the behavior of a single microlens. In this case, a $100 \times$ objective is aligned with the center of one microlens. Diaphragms with different aperture diameters are used to observe the effect of the curvature of the microlens on the AR properties. The normalized reflectivity measurements are plotted in Figure 8 for image diameters of $47 \mu \mathrm{m}, 93 \mu \mathrm{m}, 117 \mu \mathrm{m}$ and $140 \mu \mathrm{m}$. The largest aperture corresponds to nearly an entire microlens. Each curve is the average of three measurements performed on different areas of the wafer. Note that the reference is always measured with the same diaphragm size as the sample.

The curves in Figure 8 show that better attenuation, between 


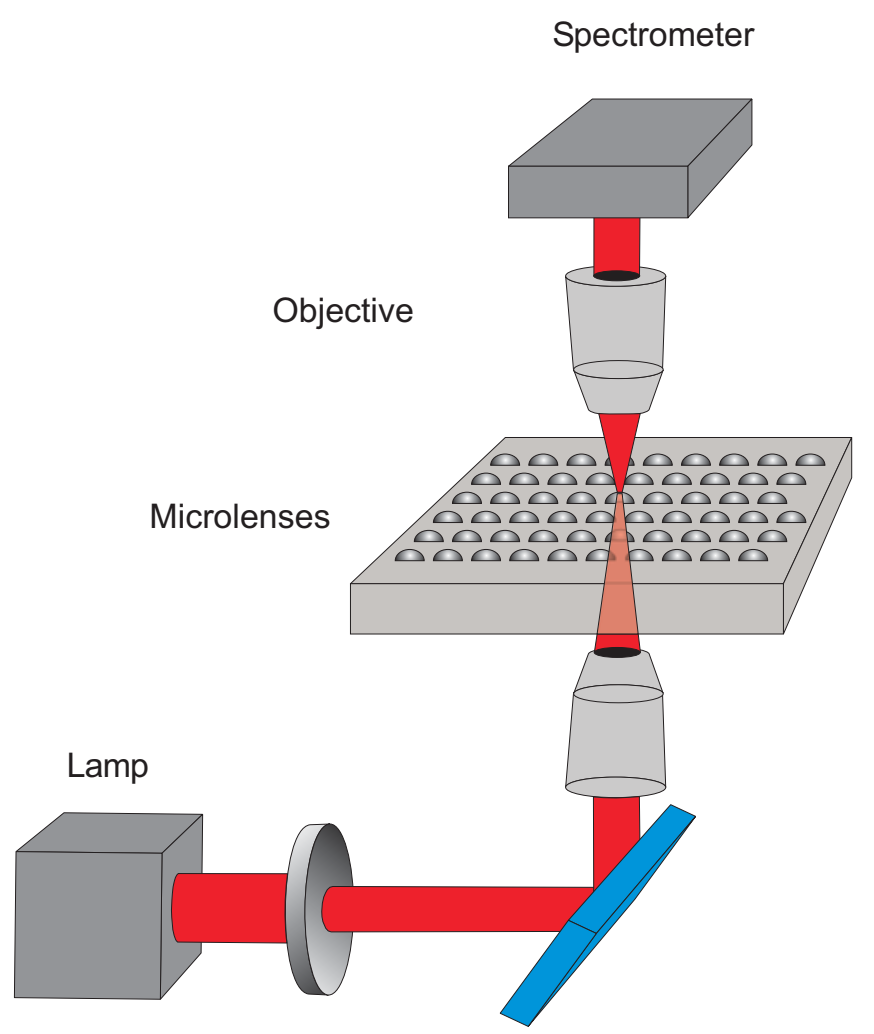

Diaphragm

FIG. 9 Setup for transmission measurements of microlenses with and without AR nanostructures.

$20 \%$ and $11 \%$, is obtained when nearly the entire microlens is considered.

Comparing Figures 6 and 8, one can see that the attenuation of the reflectivity is better (by roughly a factor of two) for the flat nanostructures than for the microlenses. This can be explained by the variation of the nanopillar's height as a function of their position on the microlens, as shown in Figure 3. An improvement of the fabrication process is necessary in order to produce a more homogeneous layer of photoresist [12].

Antireflective properties have been observed on nanostructured flat surfaces (see Figure 6) as well as on single microlenses (see Figure 8) and an area composed of several microlenses (see Figure 7). In this last case the attenuation of the reflectivity is greater than for the single lens, which shows that the flat area between the microlenses also contributes to this effect.

\section{TRANSMISSION CHARACTERIZATION}

In most cases, microlenses are used in transmission to focus light (e.g. onto a sensor array). To complete the study of our device, transmission measurements are performed.

The experimental setup used for transmission measurements is shown in Figure 9. Due to technical issues, the sample must be illuminated from backside. In order to focus light on the side of the wafer with the structure, a condenser with a numerical aperture of NA $=0.6$ is used. Light is collected by a

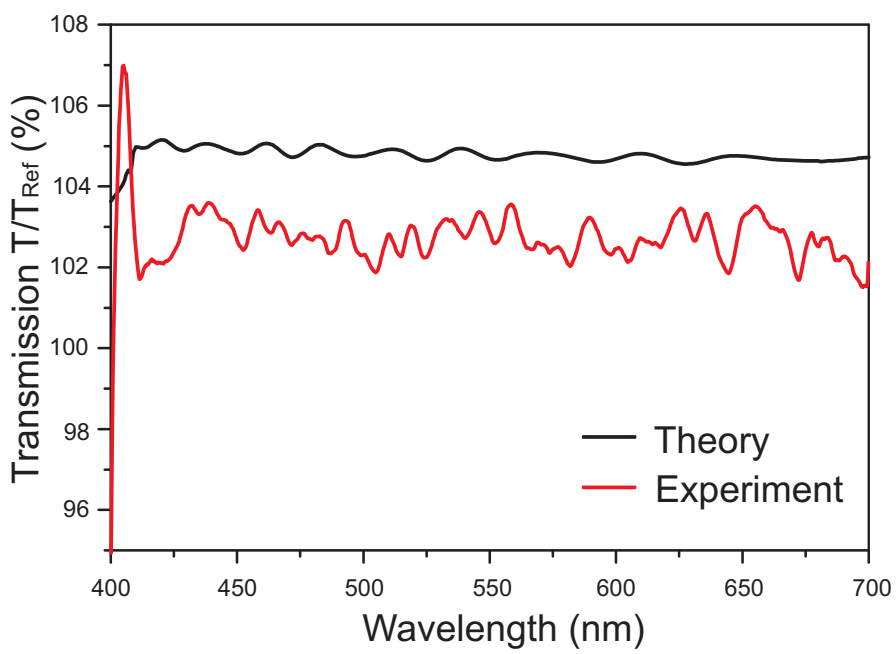

FIG. 10 The red curve shows the ratio between the transmitted light through quartz microlens with and without AR nanostructures while the black curve shows the maximum potential transmittance.

microscope objective (100×) and analyzed using a spectrometer.

Once again, the reference for the experimental measurements is a microlens without nanostructures. The normalized transmissivity curve shown in Figure 10 is the smoothed mean of three measurements taken on different areas of the wafer. The enhancement of the transmission over the visible range is $3 \%$ on average. For comparison, the maximum expected transmissivity of this device has been calculated using ray tracing simulation software FRED from Photon Engineering, (applying the Fresnel equations at the interfaces). By evaluating a microlens without nanostructures with the same illumination and collection microscope objectives (numerical apertures are taken into account) as in the experiment, we estimate the maximum transmission enhancement to be $5 \%$ according to this model.

As in the reflectivity measurements, the fabricated non-ideal nanostructures still show significant AR properties, increasing the transmissivity of the microlenses by an average of $3 \%$ across the visible spectrum. This improvement is also an appreciable fraction of the theoretical maximum enhancement of $5 \%$.

\section{TEMPERATURE STABILITY}

One of the major challenges with other approaches for AR coatings, such as multilayer thin films, is robustness to adverse conditions such as high temperatures.

In order to test the temperature stability of our AR nanostructures, transmission measurements are performed before and after heating the sample in an oven at $250^{\circ} \mathrm{C}$ for one hour and then cooling to room temperature. A $300 \mu \mathrm{m}$ area is illuminated and the transmitted light is collected by a $100 \times \mathrm{mi}-$ croscope objective (NA $=0.9$ ) using the setup shown in Figure 9. The results in Figure 11 show that the enhancement of the transmissivity stays essentially constant, which indi- 
cates that the AR nanostructure is not materially damaged by this temperature cycling. The black and red curves represent the measurements performed before and after heating respectively normalized by a reference (microlenses without nanostructures). Each curve is the average of two measurements performed on different areas of the wafer.

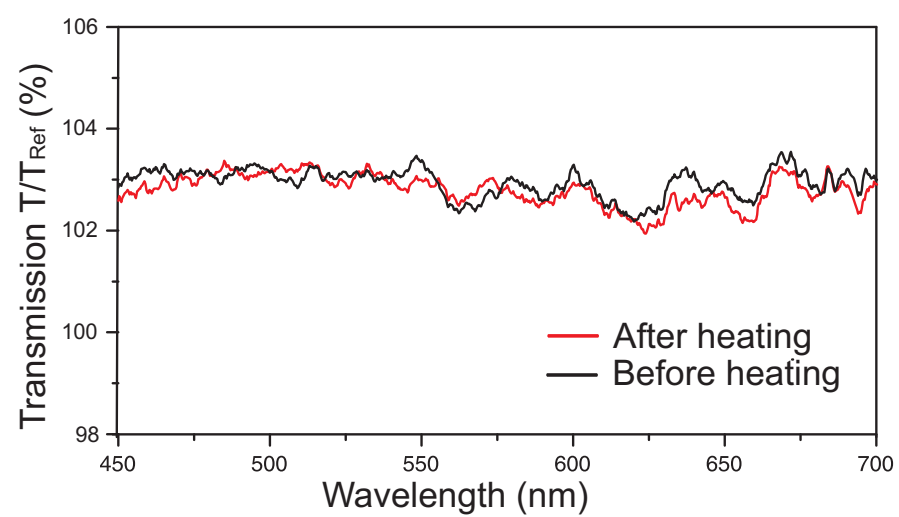

FIG. 11 Normalized transmission spectra of the nanostructured microlenses before and after baking at $250^{\circ} \mathrm{C}$ for one hour.

The durability of the AR nanostructure with respect to temperature cycling is not surprising, as the only material in the device is quartz. Moreover, as these structures are composed of a monolithic, homogenous material, they may better tolerate adverse environments, including extreme temperatures, variable humidity, immersion in liquids, or caustic surroundings. This suggests an important potential class of applications for single-material microlenses with an AR surface.

\section{CONCLUSION}

In this paper, the feasibility of fabricating an anti-reflective layer by nanostructuring large microlens arrays (4-inch wafer) has been demonstrated. The reduced reflectivity of the microlens surface is based on the engineered effective refractive index properties of a nanostructure, in this case an array of pillars that covers the entire surface of the microlenses. Although the structures on quartz microlens arrays are not perfect, the reflectivity measurements show a $15 \%$ improvement compared to unstructured microlenses. Transmission measurements, obtained with a different setup, show an enhancement of approximately $3 \%$ of the transmission. Incremental improvements in the fabrication process can further improve the performance of the antireflective surface, facilitating their incorporation into more complex micro-optical systems. An antireflective treatment of the backside of the wafer, either through nanostructuring or other methods, could also be envisaged.

In addition, we have demonstrated the interference lithography technique as a suitable method for the delicate fabrication of a nanostructure on a large, non-flat surface. This work has shown promising results consistent with theoretical predictions.

Finally, we have shown that a single-material device is stable and robust, for example to variations in temperature, which suggests that these devices may be suitable for applications involving extreme environmental conditions.

\section{References}

[1] D. Daly, R. F. Stevens, M. C. Hutley, and N. Davies, "The manufacture of microlenses by melting photoresist" Meas. Sci. Technol. 1, 759-766 (1990).

[2] Ph. Nussbaum, R. Völkel, H. P. Herzig, M. Eisner, and S. Haselbeck, "Design, fabrication and testing of microlens arrays for sensors and microsystems" Pure Appl. Opt. 6, 617-636 (1997).

[3] V. Bardinal, E. Daran, T. Leïchlé, C. Vergnenègre, C. Levallois, T. Camps, V. Conedera, J. B. Doucet, F. Carcenac, H. Ottevaere, and H. Thienpont, "Fabrication and characterization of microlens arrays using a cantilever-based spotter" Opt. Express 15, 6900-6907 (2007).

[4] S. Calixto, "Silicone microlenses and interference gratings" Appl. Opt. 41, 3355-3361 (2002).

[5] B. Päivänranta, P.-Y. Baroni, T. Scharf, W. Nakagawa, M. Kuittinen, and H. P. Herzig, "Antireflective nanostructured microlenses" Microelectron. Eng. 85, 1089-1091 (2008).

[6] M. Karlsson, and F. Nikolajeff, "Diamond micro-optics: microlenses and antireflection structured surfaces for the infrared spectral region" Opt. Express 11, 502-507 (2003).

[7] C. J. M. van Rijn, "Laser interference as a lithographic nanopatterning tool" J. Microlith. Microfab. 5, 011012 (2006).

[8] B. Päivänranta, N. Heikkilä, and M. Kuittinen, “Antireflective subwavelength-structured surfaces with enhanced color properties" J. Opt. Soc. Am. A 24, 1680-1686 (2007).

[9] T. Clausnitzer, T. Kämpfe, E.-B. Kley, A. Tünnermann, A. V. Tishchenko, and 0. Parriaux, "Highly-dispersive dielectric transmission gratings with $100 \%$ diffraction efficiency" Opt. Express $16,5577-5584$ (2008).

[10] S. Traut, M. Rossi, and H. P. Herzig, "Replicated arrays of hybrid elements for application in a low-cost micro-spectrometer array" J. Mod. Opt. 47, 2391-2397 (2000).

[11] H. Ottevaere, R. Cox, H. P. Herzig, T. Miyashita, K. Naessens, M. Taghizadeh, R. Völkel, H. J. Woo, and H. Thienpont, "Comparing glass and plastic refractive microlenses fabricated with different technologies" J. Opt. A-Pure Appl. 0p. 8, 407-429 (2006).

[12] B. Päivänranta, M. Pudas, 0. Pitkänen, K. Leinonen, M. Kuittinen, P.-Y. Baroni, T. Scharf, and H. P. Herzig, "Liquid phase deposition of polymers on arbitrary shaped surfaces and their suitability for e-beam patterning" Nanotechnology 20, 225305 (2009).

[13] P.-Y. Baroni, T. Scharf, and H. P. Herzig, "Fabrication of large-area two-dimensional photonic crystals using interference lithography and direct writing of defects" Proc. SPIE 6182, 61821V(2006).

[14] S. Traut, and H. P. Herzig, "Holographically recorded gratings on microlenses for a miniaturized spectrometer array" Opt. Eng. 39, 290-298 (2000).

[15] H. Sickinger, J. Schwider, and B. Manzke, "Fiber based MachZehnder interferometer for measuring wave aberrations of microlenses" Optik 110, 239-243 (1999).

[16] M. Born, and E. Wolf, Principles of Optics (Cambridge University Press, Cambridge, 1999).

[17] D. H. Raguin, and G. M. Morris, "Structured surfaces mimic coating performance" Laser Focus World 33, 113-117 (1997). 